\title{
Pneumologische Rehabilitation vor und nach Lungentransplantation
}

\author{
Pulmonary Rehabilitation Before and After Lung Transplantation
}

Autoren

Institut
K. Kenn, B. Sczepanski

Schön Klinik Berchtesgadener Land, Schönau a. Königssee eingereicht 13.9.2010 akzeptiert nach Revision 15. 10.2010

\section{Bibliografie}

Dol http://dx.doi.org/

10.1055/s-0030-1255938

Online-Publikation: 22.2.2011

Pneumologie 2011; 65:

419-427 @ Georg Thieme

Verlag KG Stuttgart · New York

ISSN 0934-8387

\section{Korrespondenzadresse}

Dr. Klaus Kenn

Schön Klinik

Berchtesgadener Land

Malterhöh 1

83471 Schönau a. Königssee

KKenn@schoen-kliniken.de

\section{Zusammenfassung \\ $\nabla$}

Die pneumologische Rehabilitation von Patienten mit fortgeschrittenen Lungenerkrankungen, vor allem bei COPD-Patienten, hat in den letzten Jahren an Bedeutung gewonnen. Für die Indikation Lungentransplantation (LTx) fehlen größere Studien sowohl im Hinblick auf die prä- wie auf die postoperative Rehabilitation. Dennoch finden sich zunehmend Hinweise, dass die präoperative Stabilisierung und Vorbereitung ebenso wie eine gezielte postoperative Trainingstherapie unverzichtbar zum gesamten LTx-Therapiekonzept dazugehören sollten, da der Transplantationserfolg hierdurch verbessert werden kann. Die präoperative Phase kann durch Verbesserung von Kraft, Ausdauer und Alltagsmobilität zur Überbrückung der Wartezeit genutzt werden. Zusätzlich sind intensive Motivation und Wissensvermittlung möglich, um die Patienten auf die Transplantation vorzubereiten. Ziel der postoperativen Rehabilitation ist es, mit Hilfe einer verbesserten Atemleistung die körperliche Leistungsfähigkeit und die Lebensqualität zu steigern. Dieser Beitrag stellt den Stellenwert, die Zielsetzung und die Besonderheiten einer Rehabilitation vor und nach einer Lungentransplantation dar. Abschließend wird die Bedeutung dieses perioperativen Konzepts für ein ressourcenlimitiertes und sehr kostenintensives Therapieverfahren diskutiert.

\section{Einführung}

In Deutschland werden pro Jahr knapp 250 Lungentransplantationen durchgeführt.

Idealerweise sollte der endgültigen Listung zur Transplantation eine komplexe Rehabilitation vorausgehen, um die Patienten vor der Transplantation trotz ihrer terminalen Erkrankung in einen optimalen körperlichen Zustand zu bringen und gleichzeitig alle wesentlichen Informationen zur Transplantation zu vermitteln.

\section{Abstract \\ $\nabla$}

Pulmonary rehabilitation of patients with advanced lung diseases especially with COPD patients has become more important over past years. For patients in which lung transplantation is indicated major studies concerning pre- and postoperative rehabilitation are still missing. Nevertheless, growing evidence suggests that training therapy and other components of comprehensive rehabilitation should be an essential part of the whole concept in order to augment the therapeutic success of LTx. The time prior to LTx may be used as „bridging to transplant“ by improving strength, endurance and mobility. Intensive motivation and knowledge transfer can prepare patients as good candidates. The rehabilitation after LTx aims at regaining physical abilities and health related quality of life using the increased breathing capacities. The manuscript presents the importance, aims and specific features of rehabilitation prior to and following LTx. Finally the therapeutic concept will be discussed with regard to the economic burden and the limited resources in lung transplantation.

Erste prospektiv-randomisierte kontrollierte Studien zeigen signifikante Verbesserungen durch eine solche spezialisierte Rehabilitation [1].

Ziel eines auf die Besonderheiten einer Organtransplantation ausgerichteten Rehabilitationskonzeptes ist es, neben der atemfunktionellen Optimierung auch eine Verbesserung des physischen und psychischen Befindens zu erreichen. Neben der körperlichen Rehabilitation ist die Schulung im Umgang mit allen transplantationsspezifischen Besonderheiten, insbesondere be- 


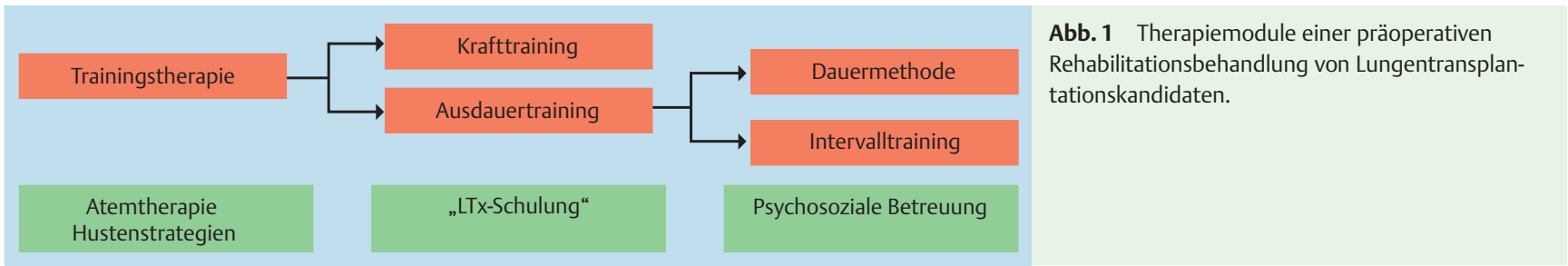

züglich Medikamenteneinnahme sowie Infekt- und Abstoßungsrealisierung von großer Bedeutung, um das bestmögliche Transplantationsergebnis zu erreichen.

Die Wiedereingliederung in den normalen Alltag, das Wiederherstellen der Selbstversorgung sowie die präoperative Vermeidung von Pflegebedürftigkeit sind wie die postoperative Wiederherstellung der Erwerbsfähigkeit wichtige Aufgaben der Rehabilitation.

Um den besonderen Bedürfnissen dieser kleinen Patientengruppe und den dazu notwendigen diagnostischen und therapeutischen Inhalten gerecht zu werden, muss die Rehabilitationseinrichtung eine besondere Infrastruktur vorhalten, die den Rahmen üblicher pneumologischer Rehabilitation deutlich übersteigt.

\section{Pneumologische Rehabilitation vor Lungen- transplantation}

In vielen Fällen erfüllen Patienten vor Lungentransplantation formell nicht mehr die gesetzlich definierten Rehabilitationsvoraussetzungen. Meist ist deren kardio-pulmonale Leistungsfähigkeit so massiv eingeschränkt, dass die Tagesaktivitäten oft nur noch aus dem essenziell Notwendigen, wie z. B. dem Gang zur Toilette, bestehen. Zur Selbstversorgung sind sie in der Regel nicht mehr in der Lage. Daher muss sich die pneumologische Rehabilitation bei Patienten im Endstadium einer Lungenerkrankung streng an den individuellen Problemen, Kapazitäten und Limitationen der Betroffenen orientieren.

\section{Angemessene Trainingsformen auswählen}

Neben einem individualisierten Ausdauertraining kommt der gezielten Steigerung der Kraft durch ein dosiertes resistives Training große Bedeutung zu. Die Steuerung des Trainings ist wichtig, da die nur geringe Leistungsfähigkeit sowohl tageszeitlichen Schwankungen wie auch äußeren Einflussfaktoren unterworfen sein kann.

Eine Hypoxämie in Ruhe oder unter Belastung ist durch eine bedarfsadaptierte Sauerstoffgabe auszugleichen. Oft ist die respiratorische Insuffizienz bei diesen Patienten derart weit fortgeschritten, dass neben einer konsequenten Sauerstoffsubstitution (LTOT) zusätzlich wegen der ausgeprägten Hyperkapnie noch eine Entlastung der überforderten Atemmuskulatur durch eine nicht-invasive Beatmung (NIV) [2-4] notwendig ist. Teilweise ist eine Trainingstherapie nur unter gleichzeitiger Anwendung von LTOT und NIV möglich [5].

Die Lebensqualität ist in diesem Erkrankungsstadium stark reduziert und erheblich davon abhängig, wie die Betroffenen gelernt haben, mit ihrer Erkrankungssituation umzugehen [6].

Abgesehen von unkontrollierten kleineren Studien aus den frühen 90er-Jahren [7] existieren noch keine prospektiven Daten zur Trainingstherapie vor LTx. Dennoch wird die perioperative Trainingstherapie bei LTx in einem aktuellen Review als ausreichend sichere Behandlung empfohlen [8].

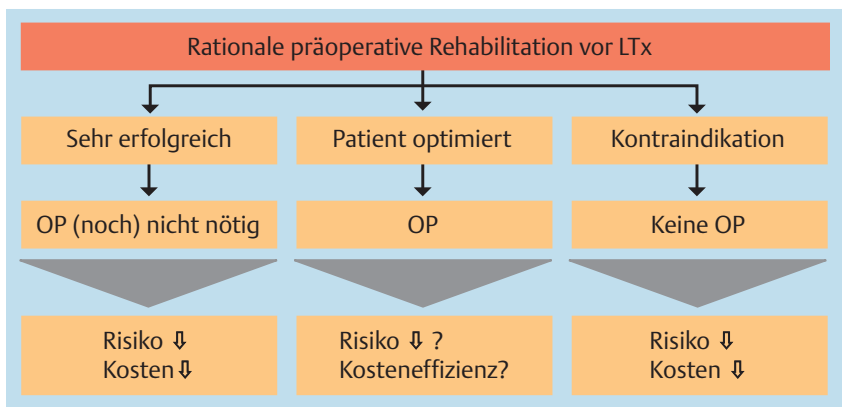

Abb. 2 Mögliche Ergebnisse einer Rehabilitation vor endgültiger Listung zur Lungentransplantation.

Verschiedene Therapieansätze im Fokus

Die Therapiemodule der Rehabilitation vor LTx sind in $\bullet$ Abb. 1 dargestellt. Neben aktiven und passiven Therapiemaßnahmen kommen inhaltlichen Aspekten wie Wissensvermittlung, Motivation, Begleitung des Qualifikations- oder Entscheidungsprozesses ebenso wie der Weitergabe von alltagsrelevantem Wissen im Umgang mit dem neuen Organ größte Bedeutung zu. In vielen Fällen ist eine psychotherapeutische Begleitung während des jeweiligen Rehabilitationsprozesses notwendig.

Die Summe aller Maßnahmen kann präoperativ einen Beitrag dazu leisten, die Selektion [9] der geeigneten Kandidaten zu verbessern, was bei der limitierten Verfügbarkeit von Spenderorganen bedeutsam ist.

\section{Unterschiedliche Ziele einer präoperativen Rehabilitation vor LTx}

Primäres Ziel einer Rehabilitation vor Lungentransplantation ist es, für die Patienten das individuell Mögliche an Stabilität und Leistungsfähigkeit zu erreichen ( $\mathbf{A}$ bb. 2). Die Optimierung aller verfügbaren konservativen Therapieansätze inkludiert sowohl das Erreichen des bestmöglichen körperlichen Leistungsvermögens als auch Strategien zum optimalen Management möglicher Exazerbationen, die oft zur bedrohlichen Destabilisierung des Gesamtbefindens führen können.

Die Ökonomisierung der Atmung durch Optimierung von Atemtechniken kann wesentlich zur Entlastung der Atemmuskulatur beitragen. Insbesondere das Erlernen von Abhusttechniken vor der Operation kann für den postoperativen Verlauf wichtig sein. Wesentlich ist für Betroffene dabei die Erkenntnis, dass diese rehabilitativen Ansätze dauerhaft eigenständig weitergeführt werden müssen. Die Wartezeit muss als Vorbereitungszeit erlebt werden.

Immer wieder finden sich Patienten, die von einer multimodalen Therapiemaßnahme derart profitieren, dass die Transplantationsdringlichkeit reduziert werden kann. Dies ist insbesondere bei COPD-Patienten an der oberen Altersgrenze für eine LTx zu beobachten, wenn durch den nicht mehr für möglich gehaltenen Zugewinn an Leistungsfähigkeit und Lebensqualität der Wunsch nach einer Transplantation in den Hintergrund tritt bzw. die er- 
Tab. 1 Inhalte und Ziele einer prä- und postoperativen gruppendynamischen „LTx-Schulung“.

$\begin{array}{ll}\begin{array}{l}\text { Schulungsinhalte prä-LTx } \\ \text { LTx-Indikationen }\end{array} & \text { Schulungsinhalte post-LTx } \\ \text { Kontraindikationen } & \text { Infekterkennung } \\ \text { Infektmanagement } & \text { akute und chronische Abstoßung } \\ \text { konsequente Nutzung LTOT (NIV) } & \text { Grundlagen des Immunsystems } \\ \text { Listungszeitpunkt } & \text { Immunsuppressiva (Neben- } \\ \text { Operationsmethoden, Über- } & \text { wirkungen, Interaktionen) } \\ \text { lebensraten nach LTx } & \text { allgemeine Lebensführung, } \\ \text { ideale Vorbereitung auf LTx } & \text { Ernährung, Hygiene und Reisen } \\ \text { Ausgestaltung der Wartezeit }= & \text { nach LTx } \\ \text { Lebenszeit } & \text { Nachsorge } \\ & \text { Neoplasie-Risiko }\end{array}$

zielten Messwerte eine aktuelle Listung nicht notwendig machen.

Eine präoperative Rehabilitation kann aufdecken, dass ein Patient für eine LTx nicht geeignet ist. Dies kann einerseits darin begründet sein, dass der Betroffene selbst in der intensiven Auseinandersetzung mit dem Thema realisiert, dass die LTx für ihn keine geeignete Therapieoption darstellt. Andererseits bietet diese längerfristige Beobachtungsphase die Möglichkeit, zuvor noch nicht realisierte Compliance-Probleme wie heimliches Rauchen oder andere in der Persönlichkeit des Einzelnen begründete Kontraindikationen zu erkennen. Vor dem Hintergrund einer aktuellen Arbeit aus Belgien, die eine ernüchternde Zahl von 11\% Rauchern unter Lungentransplantierten aufzeigt, erlangt dies besondere Bedeutung.

\section{Transplantationsspezifische Patientenschulung notwendig}

Obwohl die Transplantationszentren bereits umfangreiche Informationen zum Thema Lungentransplantation anbieten, besteht in der Regel noch ein zusätzlicher Wissens- und Aufklärungsbedarf, der in einer speziellen LTx-Gruppe, in der Patienten vor und nach LTx zusammenkommen, gedeckt werden kann. Das reale Erleben von Transplantierten sowie die dabei gegebenen gruppendynamischen Effekte können beim Entscheidungsprozess des Einzelnen hilfreich sein. Von daher sollten Patientenschulungen ein fester Bestandteil der Rehabilitation vor und nach Lungentransplantation sein. Ihnen kommt eine besondere Bedeutung zu, da sie spezielle Inhalte vermitteln, um die Eigenkompetenz des Transplantierten zu stärken und damit den Langzeiterfolg der Maßnahme zu verbessern.

Neben dem medizinischen Basiswissen rund um die Transplantation ist Therapieadhärenz bei der immunsuppressiven Therapie entscheidend für den weiteren Verlauf. Daher werden wichtige Kenntnisse z.B. über die Immunsuppressiva vermittelt (siehe - Tab.1). Eine Studie aus Hannover zeigt, dass postoperativ Non-Compliance-Probleme bestehen, z.B. eigenmächtige Dosisreduktionen in $8 \%$ oder verzögerte Einnahme bei Nebenwirkungen in $30 \%$ [10].

Daten einer unlängst veröffentlichten prospektiven Studie zum Selbstmonitoring durch Spirometrie zeigen klar den Nutzen von Schulung und Motivation auf [11,12]. Zudem müssen die Patienten lernen, dass allein anhand der Symptome (Husten, Kurzatmigkeit, FEV1-Abfall) nicht zwischen Abstoßung und Infekt unterschieden werden kann [13] und daher eine frühzeitige Vorstellung im Transplantationszentrum notwendig ist.
Alltagsrelevante Kenntnisse über notwendige Hygienemaßnahmen, sozio-familiäre Verhaltenshinweise und Reisemöglichkeiten sind bei diesen Patienten von besonderem Interesse.

Fragen, die im Zusammenhang mit Partnerschaft bzw. Kinderwunsch oder der Verhütung auftreten, können entweder Gegenstand der Gruppenschulung oder von Einzelgesprächen sein.

\section{Einbeziehung von Komorbidität in den Rehabilitationsprozess \\ $\nabla$}

\section{Ernährungsstatus meist schlecht}

Der Ernährungsstatus vieler Transplantationskandidaten ist wegen häufiger Malnutrition schlecht. Zumindest für die COPD ist bekannt, dass Untergewicht mit einem Body Mass Index (BMI) $<20$ einen unabhängigen negativen Prognosefaktor für das Überleben darstellt [14]. Somit kommt einer gut konzipierten, hoch kalorischen und nicht zu fettreichen Ernährung eine große Bedeutung zu. Die Aufteilung der Nahrung über den Tag ist von Relevanz, da zu große Magenfüllungen gerade bei COPD-Patienten zu postprandialer Atemnot führen können.

\section{Osteoporose häufig}

Eine Osteoporose kann den weiteren Verlauf nach LTx negativ beeinflussen. Diese ist meist durch systemische Steroidgaben, Immobilisation, Nikotinkonsum, hormonelle Einflüsse, chronisch inflammatorische Prozesse oder deren Kombination bedingt. So gehört die Messung der Knochendichte und falls notwendig eine optimale Osteoprotektion zum diagnostisch-therapeutischen Konzept. Bei Notwendigkeit einer dauerhaften Steroidtherapie sollte entsprechend den Leitlinien des Dachverbands Osteologie [15] bereits ab einem T-Score von $<-1,5$ eine spezifische Therapie eingeleitet werden.

\section{Depression, Angst und Panik meist verkannt und unbehandelt}

Die psychische Komorbidität bei Transplantationskandidaten ist oft nicht diagnostiziert oder wird unterschätzt. Für COPD-Patienten mindern Depression und vor allem Angst und Panikneigung nicht nur die Lebensqualität, sondern beeinflussen auch den klinischen Verlauf durch häufigere Krankenhausaufenthalte und Exazerbationen negativ. Entgegen der früheren Darstellung in der Literatur leiden die Betroffenen weniger unter Depression, als vielmehr unter Angst und Panikneigung [16]. „End of Life“Ängste, mit denen sich diese Patientengruppe nachvollziehbarerweise auseinandersetzen muss, spielen in hohem Prozentsatz eine belastende, destabilisierende Rolle [17].

Die NETT-Studie (National Emphysema Treatment Trial) zur Evaluation der operativen Lungen-Volumen-Reduktion (LVR) bei einer ähnlich schwerkranken Population von COPD-Patienten zeigte, dass ein höheres Ausmaß von Angst und Depression signifikant mit schlechterer Lebensqualität, häufigeren präoperativen Hospitalisierungen sowie einer höheren 1- und 3-Jahres-Mortalität verknüpft war [18].

In der Regel treten solche Patienten anfangs dem Gedanken an eine körperliche Rekonditionierung mit Zweifeln oder gar Ängsten entgegen. Im Verlauf weicht diese Skepsis oft dem Erleben, dass es nach langer Zeit kontinuierlicher Verschlechterung erstmals in bescheidenem Maß wieder aufwärts gehen kann.

Dies mag erklären, dass im eigenen Patientenkollektiv durch eine komplexe, multimodale Rehabilitation ohne gezielte psychothe- 
rapeutische Intervention Depression, Angst und Panikneigung vermindert werden konnten [16].

Dass Bewältigungsstrategien, im Sinne einer aktiven, positiven Auseinandersetzung der Betroffenen mit ihrer „end stage lung disease" (ESLD), zu einer deutlich besseren psychischen Lebensqualität führen können, zeigt eine Studie von Taylor [6] mit 139 COPD- und 48 CF-Patienten, die auf eine LTx warteten. Bei den COPD-Patienten dieser Untersuchung fand sich sogar eine positive Korrelation zur körperlichen Lebensqualität.

\section{Rationale für eine Trainingstherapie bei „end stage lung disease ${ }^{66}$

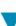

Die Ursache der regelmäßig vorliegenden Muskelatrophie ist vielschichtig. Systemische Cortisongaben, lange bestehende Immobilisation und chronisch inflammatorische Einflüsse tragen hierzu bei. Nach der Transplantation wird der Muskelabbau noch durch die Immunsuppressiva verstärkt [54]. Der für die COPD bekannte Shift vom langsamen Muskelfasertyp I zum schnellen Typ IIx ist in ähnlicher Weise auch für Lungentransplantationspatienten histologisch gesichert, bei denen neben einer Reduktion der Typ I-Fasern eine reduzierte mitochondriale oxidative Kapazität nachgewiesen wurde [19,20]. Dies führt zu einer geringeren Ausdauer und rascheren Ermüdung der peripheren Muskulatur. Die Rückführung solcher Prozesse ist langwierig und setzt gezielte Trainingsreize im Rahmen der supervidierten medizinischen Trainingstherapie voraus.

Das Heranführen an körperliche Belastung muss dabei individuell und nach vorheriger Evaluation des kardio-pulmonalen Status erfolgen. Ggf. ist anfangs nur eine minimale Intensität von wenigen Watt über kurze Zeit möglich. Am Fahrradergometer stellt das „Leertreten“, d. h. das alleinige Bewegen der Beine, für manche bereits das Maximum der Leistungsfähigkeit dar. Der Einsatz motorgetriebener Ergometer erleichtert bzw. ermöglicht vielfach erst den Einstieg in die körperliche Belastung für die Patienten.

Es finden sich zunehmend Hinweise in der Literatur, dass ein Intervalltraining bei Patienten mit erheblich limitierter Belastbarkeit einem typischen Ausdauertraining vorzuziehen ist. Dabei wird anstelle einer kontinuierlichen Belastung ein Wechsel von Belastungs- und Pausenzeiten von jeweils 30-60 Sekunden angestrebt. Bei ähnlichem Leistungszuwachs scheint das Intervalltraining mit geringerer Dyspnoe und Erschöpfung eine bessere Akzeptanz aufzuweisen [21,22].

\section{Unterschiedliche Ansätze und Ergebnisse für unterschiedliche Transplantationsindikationen? $\nabla$}

Die positiven Effekte rehabilitativer Therapieansätze sind vor allem für die COPD beschrieben. Ob Patienten mit Lungenfibrose oder Mukoviszidose einer andersartigen Trainingstherapie bedürfen, ist nicht bekannt. Für die Indikation pulmonal arterielle Hypertonie ist aufgrund der kardialen Limitation die Trainingsintensität vorsichtiger und nur unter engmaschiger klinischer und apparativer Kontrolle festzulegen [23].

Prospektive Studien müssen zeigen, ob sich die Trainingstherapie mehr an den Besonderheiten der Grundkrankheit oder generell am Schweregrad der Einschränkungen und dem Ausmaß der respiratorischen Insuffizienz orientieren muss.

\section{Indikation: COPD}

Bei COPD-Patienten liegen für die pneumologische Rehabilitation hinsichtlich positiver Effekte auf Leistungsfähigkeit, Dyspnoe, Lebensqualität, psychische Komorbidität und Reduktion von stationärer Behandlungspflichtigkeit überzeugende Daten mit höchstem Evidenzgrad vor [24 - 29]. Nur zum Teil wurden in diese Studien Patienten mit schwergradiger Erkrankung einbezogen, sodass diese Ergebnisse nicht ohne weiteres in das Vorfeld der Lungentransplantation zu übertragen sind [30]. Neu sind erste Hinweise auf eine Reduktion der Letalität durch Rehabilitationsmaßnahmen [31].

\section{Indikation: Lungenfibrose}

Günstige Ergebnisse nach Rehabilitation bei interstitiellen Lungenerkrankungen wurden erst in den letzten Jahren veröffentlicht und haben dazu geführt, dass die Rehabilitation auch bei der Lungenfibrose, nicht zuletzt wegen schwindender Evidenz medikamentöser Behandlungsansätze, zunehmend in den Fokus rückt.

Eine erste RCT-Studie zeigte bei allerdings nur geringgradig eingeschränkten Fibrosepatienten (FVC 76\% pred.) eine signifikante Verbesserung der Lebensqualität und der Gehstrecke (35 m) [32]. Ferreira [33] und Kollegen berichten über einen Zugewinn an Gehstrecke von $56 \mathrm{~m}$ bei 99 Patienten mit fortgeschrittener Lungenfibrose, von denen 66\% sauerstoffpflichtig waren. Ähnliches konnte auch für eigene Patienten mit weit fortgeschrittener Lungenfibrose unter Einschluss von Lungentransplantationskandidaten bei einer signifikanten und klinisch relevanten Verbesserung von Leistungsfähigkeit und Lebensqualität nachgewiesen werden [34].

\section{Indikation: Mukoviszidose}

Für die zystische Fibrose ist gesichert, dass die Patienten wegen der im Verlauf zunehmenden Muskelschwäche und dadurch nachlassender körperlicher Aktivität [35] von einer Rekonditionierung profitieren. Ein aktueller Cochrane-Review [36] fand von nur 26 identifizierbaren Studien lediglich 7 verwertbare Untersuchungen (mit 231 Patienten), die einen gewissen Benefit für körperliches Training bei CF-Patienten erkennen lassen, sodass die Autoren eine Trainingstherapie empfehlen.

\section{Indikation: Pulmonale Hypertonie}

Galten Patienten, die an einer relevanten chronischen pulmonalen Hypertonie litten, wegen einer befürchteten kardialen Gefährdung lange Zeit als nicht trainierbar, so konnten Mereles [23] u. Mitarb. in einer prospektiv, randomisierten, kontrollierten Studie zeigen, dass auch PAH-Patienten im höheren NYHA-Stadium $(2,9 \pm 0,5)$ bei vorhandener Expertise ohne Gefährdung bei angepasster Trainingsintensität mit einer Verbesserung von Lebensqualität und Gehstrecke (6 MWD) von $111 \mathrm{~m}$ signifikant und klinisch relevant im Vergleich zur Kontrollgruppe profitieren konnten.

\section{Andere Indikationen}

Für LTx-Indikationen wie Bronchiektasen, angeborene Fehl- oder Missbildungen, Lymphangioleiomyomatose, Histiozytosis X und andere seltene Erkrankungen finden sich keine Angaben zur Rehabilitation in der Literatur. 


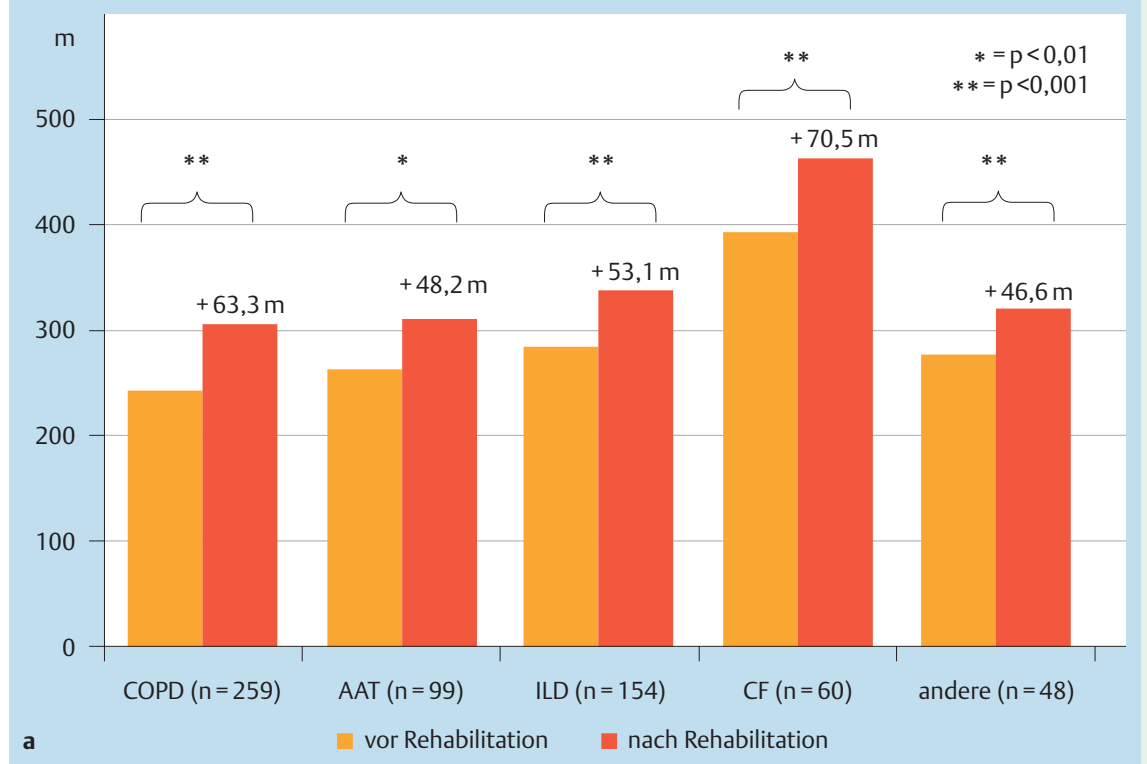

Abb. 3 a u. b Verbesserung der 6-Minuten-Gehstrecke (a) sowie der körperlichen und psychischen Summenscores der mittels SF36 gemessenen Lebensqualität (b) vor und nach präoperativer Rehabilitation von LTx-Kandidaten bei unterschiedlichen Indikationen (COPD; AAT [Alpha1-Antitrypsinmangel], ILD [Lungenfibrosen], CF [Mukoviszidose]) [37].

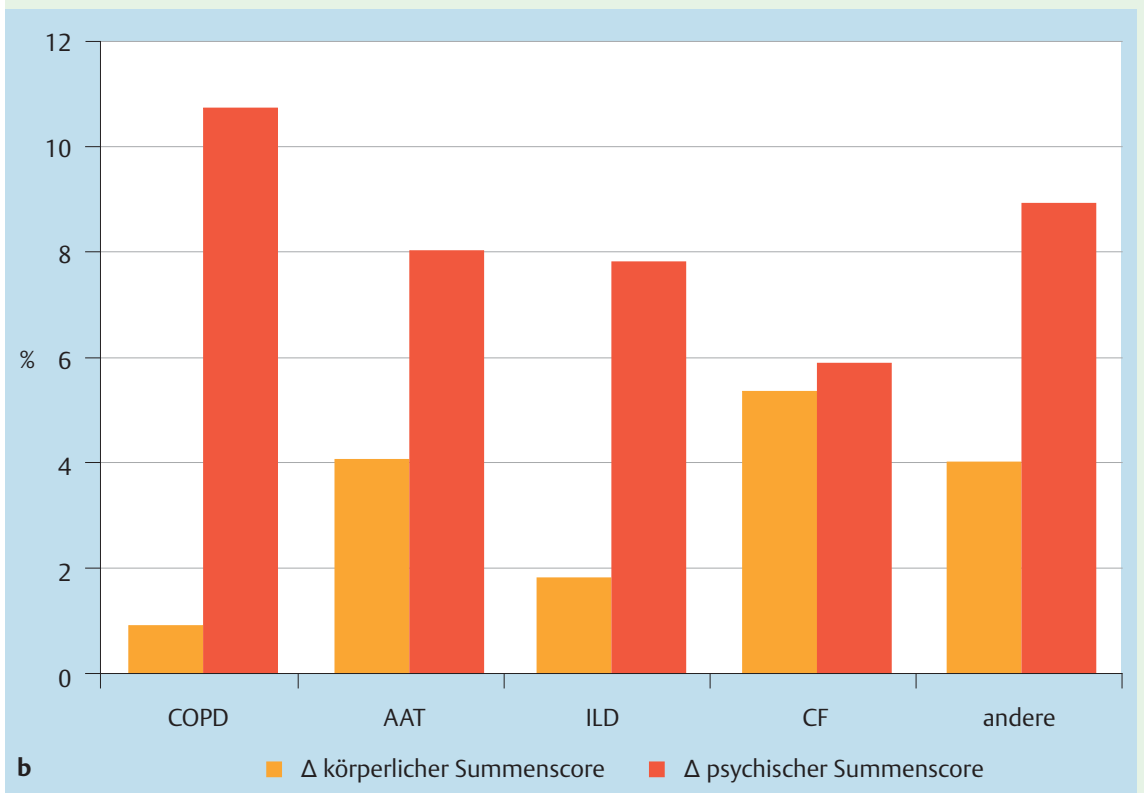

\section{Eigene Daten}

Aus einer eigenen prospektiven, offenen klinischen Studie (2000-2010) gewonnene Daten mit inzwischen mehr als 600 Transplantationskandidaten [37] zeigen sehr eindrücklich, dass durch eine multimodale Rehabilitation bei allen zur Lungentransplantation führenden Indikationen ( $\mathbf{A b b} \mathbf{3} \mathbf{3}$ u. b) signifikante, klinisch relevante Effekte zu erzielen sind.

Diese Ergebnisse sind insofern ermutigend, da mit der Evaluation für eine Transplantationsindikation bislang das Ende des üblichen Behandlungsspektrums erreicht schien.

Als minimaler, klinisch relevanter Unterschied (minimal important difference, MID) wird in der Literatur bei COPD-Patienten eine Steigerung der 6-Minuten-Gehstrecke zwischen 35 bis $54 \mathrm{~m}$ angesehen $[21,28,38,39]$. Für die Lungenfibrose wird diskutiert, dass die MID-Werte mit ca. $30 \mathrm{~m}$ deutlich niedriger liegen [32].

Ein solches Training sollte ausschließlich unter Beobachtung durch erfahrene Therapeuten erfolgen. In Einzelfällen ist Training nur in Anwesenheit eines Arztes bei gleichzeitiger Anwendung einer NIV möglich, um die eingeschränkten Leistungsreserven nicht völlig für die Atemarbeit aufzubrauchen.

Für den SF-36-Fragebogen sind für pneumologische Erkrankungen keine MIDs definiert, sodass hier eine Interpretation nur bedingt möglich ist.

\section{Resümee}

Zusammenfassend dient die präoperative Phase der Rekonditionierung, der Information und Motivation der Betroffenen. Sie zeigt ihnen auf, dass Wartezeit auch Lebenszeit ist. Die Zeit bis zur eventuellen LTx darf nicht als passives Warten, sondern muss als aktives „Trainingslager“ für diesen alles entscheidenden Eingriff begriffen werden. Die vorliegenden Daten legen nahe, dass eine Lungentransplantation nur dann in Frage kommt, wenn zuvor eine konsequente und umfassende Rehabilitation durchgeführt wird [40]. 


\section{Pneumologische Rehabilitation nach Lungentransplantation \\ $\nabla$}

Indikation und Dauer

Nach Lungentransplantation besteht ein Anspruch auf Anschlussheilbehandlung.

Eine aktuelle Arbeit aus Belgien zeigt, dass die aufgezeichnete Aktivität im ersten Jahr nach LTx im Vergleich zu einem gesunden Vergleichskollektiv trotz erfolgreicher Lungentransplantation deutlich vermindert ist [41].

Die Dauer von Rehabilitationsmaßnahmen nach LTx muss sich am Zustand des Patienten und dem Erreichen der individuell gesetzten Ziele orientieren.

Eine Rehabilitationsindikation bei LTx kann auch dann vorliegen, wenn sich im weiteren Verlauf eine allmähliche Verschlechterung des transplantierten Organs im Sinne einer chronischen Abstoßung (Bronchiolitis obliterans-Syndrom, BOS) einstellt oder andere Begleiterkrankungen zu relevanten Einschränkungen geführt haben.

Mit dem Beginn des BOS verschlechtert sich die Lebensqualität und insbesondere die körperliche Leistungsfähigkeit kontinuierlich, sodass sich daraus ein erneuter rehabilitativ-therapeutischer Ansatz ergibt [42-44], wozu allerdings erst wenige positive Daten vorliegen [45].

\section{Diagnostik im Verlauf der Rehabilitation bei LTx-Patienten}

Neben den grundlegenden Funktionsuntersuchungen wie Lungenfunktion, Blutgasanalyse und Belastungstests müssen bildgebende und endoskopische Verfahren vorgehalten werden oder unmittelbar verfügbar sein. Nur so können Verschlechterungen des Patienten durch Infekt, Abstoßung, Ergussbildung, respiratorische Insuffizienz oder Anastomosenprobleme frühzeitig detektiert werden.

Das Vorhandensein von Problemkeimen (z. B. MRSA, ESBL) beim LTx-Patient wie auch bei Patienten im Umfeld macht die Einhaltung stringenter Hygienekonzepte unverzichtbar.

Engmaschige Laborkontrollen sind hinsichtlich Infektionsscreening, CMV-Titer, Steuerung der Immunsuppressiva und deren Nebenwirkungen notwendig.

Auch nach Lungentransplantation kann es in Einzelfällen vorkommen, dass in der ersten postoperativen Phase aufgrund eines komplikationsreichen Verlaufes oder aufgrund einer ausgeprägten Atempumpenschwäche eine Sauerstoff-Langzeit-Therapie (LTOT) bzw. eine nicht-invasive Beatmung (NIV) weiterhin notwendig ist. Die Indikation ist fortlaufend zu überprüfen. Umgekehrt gestaltet sich bei einigen Patienten mitunter das Beenden einer LTOT- und/oder eine NIV-Therapie schwierig, da diese in vielen Fällen als „überlebensnotwendig“ mental fixiert sind.

\section{Osteoporose beachten}

Neben den bereits genannten Osteoporoseursachen in der präLTx-Phase kommt es postoperativ durch die Immunsuppressiva zu einem verstärkten Knochenabbau. Die Inzidenz der Osteoporose in diesem Kollektiv ist hoch und stellt eine relevante Komorbidität dar. Trotz umfangreicher präoperativer Diagnostik ist die Knochendichte bei bis zu einem Fünftel der Transplantierten nicht bekannt bzw. nicht optimal therapiert [46]. Es gibt deutliche Hinweise dafür, dass körperliches Training beim Lungentransplantierten in Kombination mit einem Bisphosphonat die Knochendichte stärker verbessert als ein Bisphosphonat allein [47].

\section{Psychotherapiebedarf}

Der psychologische Betreuungsbedarf nach Lungentransplantation ist erheblich. Daher sollten erfahrene Psychotherapeuten den besonderen Bedürfnissen von Transplantierten Rechnung tragen.

Trotz erfolgter Transplantation werden von den Patienten verbliebene körperliche Beeinträchtigungen als Frustrationen erlebt und beeinträchtigen die Lebensqualität. Andererseits kann auch der Wegfall von Hilfsbedürftigkeit und Abhängigkeit und das dadurch veränderte sozio-familiäre Rollenverständnis Anlass zu Gesprächsbedarf geben. Themen wie Zukunftsplanung und die Angst vor einer möglichen Abstoßung spielen ebenso eine Rolle. Der selbstbewusste Umgang mit transplantationsspezifischen Notwendigkeiten (Ernährungsgewohnheiten, Mundschutz, Hygiene, soziale Kontakte, Partnerschaft, etc.) ist zu erlernen. Dabei kann der Austausch mit anderen Transplantierten von immenser Bedeutung sein. Bei Bedarf werden auch Angehörige in den Gesamtprozess einbezogen.

Im Langzeitverlauf ist psychologische Begleitung insbesondere dann sinnvoll, wenn sich durch ein BOS eine erneute allmähliche Verschlechterung einstellt [48] und dadurch dem Patienten ein erneutes, langsames Organversagen bewusst wird.

\section{Ernährungsberatung und Schulung}

Je nach Ernährungsstatus sind entweder kalorienreiche oder kalorienreduzierte Empfehlungen auszusprechen. Die Messung von body-composition oder Bio-Impedanz kann helfen, die Veränderungen der Körperzusammensetzung exakt zu erfassen und damit den Patienten zu gezielten Ernährungsinterventionen zu motivieren.

Bei der Ernährungsberatung sind neben dem gastro-ösophagealen Reflux und der Gastroparese häufig medikamentös-induzierte diabetische Stoffwechsellagen zu beachten. Eine Hyperlipidämie ebenso wie eine Osteoporose sind bei Beratung und Kosterstellung zu berücksichtigen [49].

Die Aufnahme von Krankheitserregern über Nahrungsmittel sowie mögliche Interaktionen von Lebensmitteln mit Medikamenten (z.B. Grapefruitsaft/Immunsuppressiva) sind weitere Schulungsinhalte.

\section{Physiotherapie}

Ziel der Atemphysiotherapie nach Lungentransplantation ist die weitere Verbesserung von Ventilation und Sekretelimination. Vorbestehende Defizite der Atmung durch die Grunderkrankung werden postoperativ durch mögliche Folgen von Intubation, (Langzeit-)Beatmung, in seltenen Fällen unter Einschluss eines Tracheostomas und nicht zuletzt durch die eingeschränkte postoperative Mobilität verstärkt. Operationsbedingt können Schmerzen, Pleuraergüsse und Zwerchfellparesen die Ventilation beeinträchtigen.

Narbenbehandlungen und physiotherapeutische Maßnahmen zur Verbesserung der Atemwahrnehmung und der Zwerchfellaktivität sind neben einer Mobilisation oft starrer Thoraxstrukturen notwendig, um eine Atemvertiefung und damit eine Steigerung der Vitalkapazität zu erreichen. Einige Patienten haben aus der präoperativen Phase dyspnoebedingte Atemmuster mit erhöhter Atemfrequenz engrammiert, die schrittweise „abtrainiert" werden müssen.

Die verminderte mukoziliäre Clearance ist durch die Denervation der transplantierten Lunge mit Verlust des Hustenreflexes beeinträchtigt [50]. Der dadurch weniger effektive Hustenstoß kann zu einer erhöhten Infektionsneigung führen. Hier können spezielle 
Hustentechniken (z. B. Huffing, autogene Drainage) in Kombination mit Atemhilfsgeräten, flankiert von einer adäquaten Schmerztherapie, die Husteneffektivität erhöhen [51]. Während sich der Hustenreflex vermutlich im Laufe des ersten Jahres erholen kann [52], bleibt die mukoziliäre Clearance dauerhaft gestört [53].

Evidenzbasierte Daten zur Atemphysiotherapie bei Lungentransplantation liegen nicht vor.

Bestehende psychomotorische Defizite [54] können durch ergotherapeutische Behandlung der Feinmotorik, supervidierte Gehund Treppensteigübungen und ggf. computergestütztes Hirnleistungstraining gebessert werden. Zusätzlich kann das Training von Alltagsbelastungen (ADL-Training) hilfreich sein.

\section{Neue Lunge ausreichend? - Nur über gezieltes Training zum maximalen Erfolg}

Das gravierendste präoperative Problem, die Atemnot, wird durch die Transplantation meist beseitigt. Es persistiert aber dennoch oft eine eingeschränkte körperliche Leistungsfähigkeit.

Befragt man Lungentransplantierte postoperativ hinsichtlich subjektiv empfundener Lebensqualität und Leistungsfähigkeit, so unterscheidet sich die Einschätzung kaum von der einer altersgleichen gesunden Kontrollgruppe [55,56]. Werden jedoch objektive Leistungsparameter betrachtet, zeigt sich ein deutlich niedrigeres Niveau der körperlichen Leistungsfähigkeit im Vergleich zur Kontrollgruppe (maximale Leistung 65 versus 195 Watt) und somit eine deutliche Überschätzung der eigenen körperlichen Belastbarkeit der Transplantierten [57].

Einige Studien zeigen, dass mit normaler Alltagsaktivität (ADL) das Maximum an körperlicher Leistungsfähigkeit noch nicht erreicht wird [58]. Eine konsequente Trainingstherapie hingegen scheint die Leistungsfähigkeit (6 MWD) im perioperativen Verlauf zu optimieren [55].

Einige neuere Studien bestätigen diese Erfolge. Belgische Kollegen [59] zeigen mit unseren Daten vergleichbare Ergebnisse einer Trainingstherapie nach LTx (6 MWD +140 m), wohingegen in der australischen Studie [60], die nur selektierte Patienten mit komplikationslosem Verlauf für ihr ambulantes Setting zuließen, auffallend hohe 6MGT-Resultate gezeigt wurden.

Ein aktueller Übersichtsartikel zur Trainingstherapie bei Lungentransplantierten betont das Fehlen belastbarer Daten, sodass zwar positive Effekte auf Lebensqualität, körperliche Belastbarkeit, Muskelfunktion und Knochendichte beschrieben werden, allgemeine Empfehlungen für Art, Häufigkeit und Dauer jedoch nicht gegeben werden.

Eigene Daten von inzwischen mehr als 530 Transplantierten unseres Zentrums aus der frühen postoperativen Phase $(<1 \mathrm{Jahr})$ zeigen nach multidisziplinärer Rehabilitation Gehstreckenverbesserungen von ca. $116 \mathrm{~m}$ in 6 Minuten ( $\bullet$ Abb.4), was sich auch in einer deutlichen Steigerung der Lebensqualität (SF36) niederschlägt [61].

\section{Sozialmedizinische Beurteilung und Sozialberatung}

Die sozialmedizinische Begutachtung im Rahmen der postoperativen LTx-Rehabilitation kann nach eigener Erfahrung aufgrund des noch vorhandenen weiteren Genesungspotenzials nur bedingt vorgenommen werden. Diesem Umstand trägt die Sozialgesetzgebung mit dem Begriff der Heilungsbewährung (ähnlich wie bei Tumorerkrankungen) Rechnung. Nach einer LTx wird meist für zwei Jahre ein Grad der Behinderung (GdB) von 100\% angesetzt. Unter Berücksichtigung der Immunsuppression bleibt

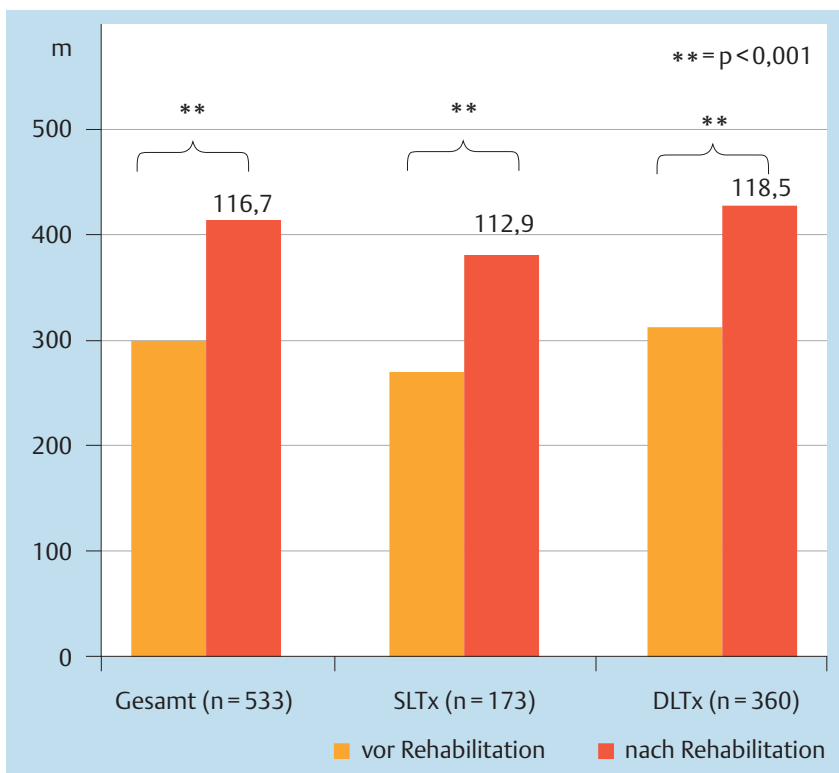

Abb. 4 Verbesserung der 6-Minuten-Gehstrecke bei Lungentransplantierten vor und nach postoperativer Rehabilitation. Die Ergebnisse nach Einzellungentransplantation (SLTx) und Doppellungentransplantation (DLTx) sind vergleichbar (51).

danach der GdB selbst bei günstigem Heilungsverlauf bei mindestens $70 \%[62]$.

Nach komplikationsreichen Verläufen kann eine Sozialberatung bezüglich des Grads der Behinderung oder Pflegestufen notwendig sein. Die Beantragung von Leistungen zur Teilhabe am Arbeitsleben und die Beratung zur Wiedereingliederung in das Berufsleben sind für das Rehabilitationsziel der beruflichen und sozialen (Re-)Integration wichtig.

In einer aktuellen Studie der MH Hannover wird der Prozentsatz von Teil- oder Vollzeitbeschäftigten für diese Klientel im Langzeitverlauf mit 28,7 nach 1 Jahr und 7,4\% nach 5 Jahren angegeben [63].

Die Wahrscheinlichkeit wieder ins Berufsleben zurückzukehren, steigt mit jüngerem Alter, guter Lebensqualität und einer Berufstätigkeit vor der Transplantation [64,65].

\section{Fazit - Perioperative Rehabilitation bei LTx - ein hochselektiver Anspruch \\ $\nabla$}

Zusammenfassend stellt das Verfahren einer Lungentransplantation von der Indikationsstellung über die Durchführung bis zur Nachbetreuung modellhaft eine außerordentlich komplexe Therapieoption dar, die die Implementierung einer kompetenten, qualitativ hochwertigen und maßgeschneiderten perioperativen Rehabilitation notwendig erscheinen lässt. In den USA, einem Land, in dem sonst die Rehabilitation eine deutlich geringere Rolle spielt, ist eine perioperative Trainingstherapie im Rahmen einer Lungentransplantation nicht nur unverzichtbar, sondern für einzelne Zentren sogar eine „conditio sine qua non“ [55].

Die präoperative Vorbereitung dient nicht ausschließlich der Rekonditionierung und dem gezielten Hinarbeiten auf eine Lungentransplantation, sondern kann auch Erkenntnisse hinsichtlich Kontraindikationen und Patientenselektion erbringen. In einigen Fällen kann der Erfolg der Maßnahme zum Aufschub der Dringlichkeit oder mitunter sogar zur Aufhebung der LTx-Indikation führen. 
Postoperativ kann eine hochwertige Rehabilitation zu einem Optimum an Leistungsfähigkeit, Lebensqualität und Eigenkompetenz der Patienten beitragen.

Prospektive Studien werden zeigen müssen, ob eine perioperative Rehabilitation bei Lungentransplantation die dargestellten Ziele tatsächlich erreichen kann.

Die limitierte Verfügbarkeit von Organen, die enorm hohen Kosten einer Lungentransplantation für das Gesundheitssystem und vor allem die lebensentscheidende Bedeutung des Transplantationserfolgs für den einzelnen Patienten rechtfertigen diesen besonderen Aufwand.

\begin{tabular}{|c|c|}
\hline \multicolumn{2}{|c|}{$\begin{array}{l}\text { Abkürzungen } \\
\nabla\end{array}$} \\
\hline 6MWD & $\begin{array}{l}6 \text { Minute Walk Distance } \\
\text { 6-Minuten-Gehtest }\end{array}$ \\
\hline ADL & $\begin{array}{l}\text { Activities of Daily Living } \\
\text { Alltagsaktivitäten }\end{array}$ \\
\hline BMI & Body Mass Index \\
\hline DLTx & $\begin{array}{l}\text { Double Lung Transplantation } \\
\text { Doppellungentransplantation }\end{array}$ \\
\hline HRQL & $\begin{array}{l}\text { Health Related Quality of Life } \\
\text { Lebensqualität }\end{array}$ \\
\hline LTOT & $\begin{array}{l}\text { Long Term Oxygen Therapy } \\
\text { Langzeitsauerstofftherapie }\end{array}$ \\
\hline LTX & $\begin{array}{l}\text { Lung Transplantation } \\
\text { Lungentransplantation }\end{array}$ \\
\hline LVR & $\begin{array}{l}\text { Lung Volume Reduction Surgery } \\
\text { Lungenvolumenreduktion }\end{array}$ \\
\hline NIV & $\begin{array}{l}\text { Non Invasive Ventilation } \\
\text { nicht invasive Beatmung }\end{array}$ \\
\hline PAH & Pulmonal Arterial Hypertonie \\
\hline $\mathrm{RCT}$ & Randomized Controlled Trial \\
\hline SLTx & $\begin{array}{l}\text { Single Lung Transplantation } \\
\text { Einzellungentransplantation }\end{array}$ \\
\hline
\end{tabular}

\section{Interessenkonflikt}

$\nabla$

Die Autoren geben an, dass kein Interessenkonflikt besteht.

\section{Literatur}

1 Langer $D$ et al. Effects of exercise training after lung transplantation: preliminary results of a randomized controlled trial. Am J Respir Crit Care Med 2010; 181: A1206

2 Garrod R, Mikelsons C, Paul EA et al. Randomised controlled trail of domiciliary non-invasive positive pressure ventilation and physical training in severe chronic obstructive pulmonary disease. Am J Respir Crit Care Med 2000; 162: 1335-1341

3 Kenn K, Schoenheit-Kenn U, Bösl T et al. Effects of pulmonary rehabilitation including exercise training in patients with indication for noninvasive ventilation therapy. AJRCCM 2002; 165: A735

4 Köhnlein T, Schönheit-Kenn U, Winterkamp S et al. Noninvasive ventilation in pulmonary rehabilitation of COPD patients. Respiratory Medicine 2009; 103: 1329-1336

5 Schoenheit-Kenn U, Winterkamp S, Boensch $M$ et al. Effects of pulmonary rehabilitation including exercise training in COPD Patients with indication for non-invasive ventilation therapy (NIV). AJRCCM 2009; 179: A1053

6 Taylor JL et al. Coping and quality of life in patients awaiting lung transplantation. J of Psychosomatic Research 2008; 65: 71 - 79

7 Biggar D, Trulock E, Patterson G, Cooper J. Medium term results of pulmonary rehabilitation prior to lung transplantation (abstract). Am Rev Respir Dis 1993; 147: A333
8 Mathur S, Hornblower E, Levy RD. Exercise training before and after lung transplantation. Phys Sportsmed 2009; 37: 78-87

9 Gottlieb J. Update on lung transplantation (Review). Therapeutic Advances in Respiratory disease 2008; 2: 237-247

10 Kugler C, Fischer S, Gottlieb J et al. Symptom experience after lung transplantation: impact on quality of life and adherence. Clin Transplant 2007; 21: $590-596$

11 Kugler C, Fuehner T, Dierich $M$ et al. Effect of adherence to home spirometry on bronchiolitis obliterans and graft survival after lung transplantation. Transplantation 2009; 88: 129-134

12 DeVito Dabbs A, Dew MA, Myers B et al. Evaluation of a hand-held, computer-based intervention to promote early self-care behaviors after lung transplant. Clin Transplant 2009; 23: 537-545

13 DeVito Dabbs A, Hoffman LA, Iacono AT et al. Are symptom reports useful for differentiating between acute rejection and pulmonary infection after lung transplantation? Heart Lung 2004; 33: 372 - 380

14 Schols AMWJ, Slangen J, Volovics L, Wouters EFM. Weight loss is a reversible factor in the prognosis of chronic obstructive pulmonary disease. Am J Respir Crit Care Med 1998; 157: 1791 - 1797

15 Dachverband Osteologie. DVO-Leitlinie 2009 zur Prophylaxe, Diagnostik und Therapie der Osteoporose bei Erwachsenen. Osteologie 2009; 18: $304-328$

16 Kühl K, Kuhn C, Kenn K, Rief W. Der COPD-Angst-Fragebogen (CAF): Ein neues Instrument zur Erfassung krankheitsspezifischer Ängste bei COPD-Patienten. Psychother Psychosom Med Psychol 16 Apr 2010 [Epub ahead of print]

17 Curtis JR, Engelberg RA, Nielsen EL et al. Patient-physician communication about end-of-life care for patients with severe COPD. Eur Respir J 2004; 24: 200-205

18 Fan VS. Sex, depression and risk of hospitalization and mortality in COPD. Arch Intern Med 2007; 167: 2345-2353

19 Krieger AC, Szidon P, Kesten S. Skeletal muscle dysfunction in lung transplantation. J Heart Lung Transplant 2000; 19: 392-400

20 Wang XN, Williams TJ, McKenna MJ et al. Skeletal muscle oxidative capacity, fiber type, and metabolites after lungtransplantation. Am J Respir Crit Care Med 1999; 160: 57-63

21 Vogiatzis I, Terzis G, Nanas $S$ et al. Skeletal muscle adaptations to interval training in patients with advanced COPD. Chest 2005; 128: 3838 3845

22 Glöckl R, Weber-Lange B, Halle M, Kenn K. Ausdauertraining bei endgradiger Lungenerkrankung vor Lungentransplantation - welche Trainingsform ist geeignet? Pneumologie 2010; 64: V251; DOI: 10.1055/s-0030-1251090

23 Mereles D, Ehlken N, Kreuscher S et al. Exercise and respiratory training improve exercise capacity and quality of life in patients with severe chronic pulmonary hypertension. Circulation 2006; 114: 1448-1449

24 Ries AL, Bauldoff GS, Carlin BW et al. Pulmonary rehabilitation executive summary. Joint American College of Chest Physicians/American Association of Cardiovascular and Pulmonary Rehabilitation Evidence-Based Clinical Practice Guidelines. Chest 2007; 131: 1S - 3S

25 Nici L, Raskin J, Rochester CL et al. Pulmonary rehabilitation: What we know and what we need to know. J Cardiopulm Rehabil Prev 2009; 29: $141-151$

26 Celli BR. Update on the management of COPD. Chest 2008; 133: 1451 1462

27 Troosters T, Casaburi R, Gosselink $R$ et al. Pulmonary rehabilitation in chronic obstructive pulmonary disease. Am J Respir Crit Care Med 2005; $172: 19-38$

28 Lacasse Y, Goldstein R, Lasserson TJ et al. Pulmonary rehabilitation for chronic obstructive pulmonary disease. Cochrane database of Systematic Reviews 2006; 4: CD003793; DOI: 10.1002/14651858.CD003793. pub2

29 Casaburi $R, Z u$ Wallack R. Pulmonary Rehabilitation for management of chronic obstructive pulmonary disease. N Engl J Med 2009; 360: $1329-1335$

30 Ambrosino N, Simonds A. The clinical management of extremely severe COPD. Respiratory Medicine 2007; 101: 1613-1624

31 Puhan MA, Gimeno-Santos E, Scharplatz M et al. Pulmonary rehabilitation following exacerbations of chronic obstructive pulmonary disease. Cochrane database of Systematic Reviews 2009; 1: CD005305; DOI: 10.1002/14651858.CD005305.pub2

32 Holland AE et al. Small changes in six-minute walk distance are important in diffuse parenchymal lung disease. Respiratory Medicine 2009; 103: $1430-1435$ 
33 Ferreira A, Garvey C, Connors GL et al. Pulmonary rehabilitation in interstitial lung disease: benefits and predictors of response. Chest 2009; 135: $442-447$

34 Kaufmann $U$, Boensch M, Studnicka M, Kenn K. Effects of pulmonary rehabilitation in patients with interstitial lung disease (ILD). ERSJ 2009; 34 (Suppl. 53): 286s

35 Troosters T, Langer D, Vrijsen B et al. Skeletal muscle weakness, exercise tolerance and physical activity in adults with cystic fibrosis. Eur Respir J 2009; 33: 99-106

36 Bradley J, Moran F. Physical training for cystic fibrosis. Cochrane Database Syst Rev 2008; 23: CD002768

37 Kenn K, Winterkamp S, Schoenheit-Kenn et al. Effects of pulmonary rehabilitation prior to lung transplantation (LTx) in patients with end stage lung diseases. AJRCCM 2009; 179: A5381

38 Redelmeier DA, Bayoumi AM, Goldstein RS et al. Interpreting small differences in functional status: the Six Minute Walk test in chronic lung disease patients. Am J Respir Crit Care Med 1997; 155: 1278 1282

39 Puhan MA, Mador MJ, Held $U$ et al. Interpretation of treatment changes in 6-minute walk distance in patients with COPD. Eur Resp J 2008; 32 : 637-643

40 Decramer $M$. Treatment of chronic respiratory failure: lung volume reduction surgery versus rehabilitation. Eur Respir J 2003; 22 (Suppl. 47): $47-56$

41 Langer D, Gosselink R, Pitta F et al. Physical activity in daily life 1 year after lung transplantation. Heart Lung Transplant 2009; 28: 572-578

42 Vermeulen KM, Groen H, van der Bij W et al. The effect of bronchiolitis obliterans syndrome on health related quality of life. Clin Transplant 2004; 18: 377-383

43 Vermeulen KM, Ouwens JP, van der Bij W et al. Long-term quality of life in patients surviving at least 55 months after lung transplantation. General Hospital Psychiatry 2003; 25: $95-102$

44 van den Berg J, Geertsma A, van der Bij W et al. Bronchiolitis obliterans syndrome after lung transplantation and health-related quality of life. Am J Respir Crit Care Med 2000; 161: 1937-1941

45 Kenn K, Sczepanski B, Weber-Lange B et al. Effects of pulmonary rehabilitation performed more than one year after lung transplantation. AJRCCM 2009; 179: A5376

46 Sczepanski B, Schwarzl G, Winterkamp S et al. Knochendichte nach Lungentransplantation: Messungen kurz nach OP und in der Langzeitbetrachtung. Pneumologie 2010; 64: P308; DOI: 10.1055/s-00301251304

47 Braith RW, Conner JA, Fulton MN et al. Comparison of alendronate vs alendronate plus mechanical loading as prophylaxis for osteoporosis in lung transplant recipients: a pilot study. J Heart Lung Transplant 2007; 26: $132-137$

48 Künsebeck HW, Kugler C, Fischer S et al. Quality of life and bronchiolitis obliterans syndrome in patients after lung transplantation. Prog Transplant 2007; 17: $136-141$

49 Tynan C, Hasse JM. Current nutrition practices in adult lung transplantation. Nutr Clin Pract 2004; 19: 587 - 596

50 Dolovich M, Rossman C, Chambers $C$ et al. Mucociliary function in patients following single lung or Iung/heart transplantation. Am Rev Respir Dis 1987; 135: 363

51 Downs AM. Physical therapy in lung transplantation. Phys Ther 1996; 76: $626-642$
52 Duarte A, Terminella L, Smith J et al. Restoration of Cough Reflex in Lung Transplant Recipients. Chest 2008; 134: 310-316

53 Herve P, Silbert D, Cerrina J et al. Impairment of bronchial mucociliary clearance in long-term survivors of heart/lung and double-lung transplantation. The Paris-Sud Lung Transplant Group. Chest 1993; 103 : $59-63$

54 Wells CL, Smiley-Oyen AL, Dauber JH. Psychomotor performance in lung transplant recipients: simple reaction time. J Heart Lung Transplant 2005; 24: $282-288$

55 Gaissert HA, Cooper JD, Pohl MS, Patterson GA. Comparison of early functional results after lung volume reduction or lung transplantation for chronic obstructive pulmonary disease. J Thorac Cardiovasc Surg 1996; 111: 296-306

56 Smeritschnig B, Jaksch P, Kocher A et al. Quality of life after lung transplantation: a cross-sectional study. The Journal of Heart and Lung transplantation 2005; 24: 474-480

57 Tegtbur U, Sievers C, Busse MW et al. Lebensqualität und körperliche Leistungsfähigkeit bei Patienten nach Lungentransplantation. Pneumologie 2004; 58: 72 - 78

58 Stiebellehner L, Quittan M, End A et al. Aerobic endurance training program improves exercise performance in lung transplant recipients. Chest 1998; 113: 906-912

59 Maury G, Langer D, Verleden G et al. Skeletal muscle force and functional exercise tolerance before and after lung transplantation: a cohort study. Am J Transplant 2008; 8: 1275 - 1281

60 Munro PE, Holland AE, Bailey $M$ et al. Pulmonary rehabilitation following lung transplantation. Transplant Proc 2009; 41: 292 - 295

61 Sczepanski B, Bönsch M, Kenn K. Effekte einer pneumologischen Rehabilitation in einem spezialisierten Zentrum nach Einzel- oder Doppellungentransplantation. Pneumologie 2010; 64: V310; DOI: 10.1055/s0030-1251092

62 Anlage zu $\S 2$ Versorgungsmedizin-Verordnung - VersMedV, vom 10. Dezember 2008, Bundesgesetzblatt Jahrgang 2008 Teil I Nr. 57 www.bgbl.de; Stand: 12.9.2010

63 Kugler C, Tegtbur U, Gottlieb J et al. Health-related quality of life in longterm survivors after heart and lung transplantation: a prospective cohort study. Transplantation 2010; 90: 451-457

64 Petrucci L, Ricotti S, Michelini I et al. Return to work after thoracic organ transplantation in a clinically-stable population. Eur J Heart Fail 2007; 9: $1112-1119$

65 Cicutto L, Braidy C, Moloney S. Factors affecting attainment of paid employment after lung transplantation. J Heart Lung Transplant 2004; 23: $481-486$

\section{Bisher erschienene Beiträge dieser Serie \\ $\nabla$}

1 Schreder $T$ et al. Lungentransplantation bei Lungenemphysem - Wer? Wann? Wie? Pneumologie 2010; 64: 632-639

2 Neurohr $C$ et al. Immunsuppression und Infektionsprophylaxe nach Lungentransplantation. Pneumologie 2011; 65: 94-102

3 Kroegel $\mathrm{C}$ et al. Atemwegskomplikationen nach Lungentransplantation - Klinik, Diagnose und interventionelle Behandlung. Pneumologie 2011; 65: 293-307 